\title{
Beyond folk theories of CSCL
}

\author{
Gerry Stahl • Friedrich Hesse
}

Published online: 16 September 2010

(C) International Society of the Learning Sciences, Inc.; Springer Science+Business Media, LLC 2010

The role of theory in CSCL research is a complex matter, which has not been well worked out yet. The short caricature is this: There are three kinds of researchers in the field of CSCL,

1. People who conduct CSCL research and report on it as though there is no need for theory; they simply observe results of interventions.

2. People who conduct CSCL research and report on it by following a particular theory or theoretical framework that they accept as is.

3. People who conduct CSCL research in order to investigate theoretical issues and refine theoretical perspectives specific to CSCL.

The first group of people is naïve. The philosophy of science has shown convincingly that research is necessarily theory-laden. Those who do not reflect on their theoretical footing simply adopt the assumptions of common sense, known as 'folk theories'. Folk theories are based on experiences of everyday life, on distinctions embedded in common language, and on simplifications of outmoded theories. For instance, folk theories might assume that what a subject says in an interview or a survey directly represents what the researcher was looking for, without worrying about how the imposed situation might influence the subject's response or the researcher's interpretation.

The second group may be much more sophisticated about research methodology, having learned from established sciences like psychology, education, linguistics, or informatics. They are skilled at setting up survey instruments, research designs, and statistical analyses. They are also adept at critically evaluating each other's claims. Using the constructs of a given theoretical framework, researchers in this group like to test theoretical predictions, for instance to see if a specific educational intervention will increase student learning outcomes as measured by gains from pre-test to post-test. While findings from such an

\footnotetext{
G. Stahl $(\bowtie)$

Drexel University, Philadelphia, PA, USA

e-mail: Gerry.Stahl@drexel.edu

F. Hesse

Knowledge Media Research Center, Tubingen, Germany

e-mail: f.hesse@iwm-kmrc.de
} 
approach can be useful, the limitation is that the imposition of a theory that was not explicitly developed for CSCL may fail to identify phenomena that are characteristic of CSCL-and which could therefore be of particular interest to people involved in implementing CSCL in practice.

The third group may start from a stated theoretical framework or even from a commonsensical understanding - for all thought is necessarily grounded in everyday language and in the tacit pre-understandings that come from human activity in the world. However, their research aims at pushing the theories further and refining the conceptualizations through which collaborative learning is comprehended. The articles in this issue of ijCSCL exemplify such an approach.

The sciences in which many CSCL researchers were trained generally focus on the individual as the subject, who learns. Often, these sciences recognize the influence of cultural and historical influences, but these are generally conceived of at a broad societal level. In contrast, CSCL settings typically involve processes (cognitive, knowledge-building, interactional, or identity-forming) at the small-group and/or classroom level of description, as well as at the individual student level. Processes at these different levels interpenetrate with each other intimately, without being reducible to any one level. In addition, CSCL involves mediation of the learning, interaction, and cognition by technological artifacts and computational media. To capture these processes and mediations, researchers need to develop more elaborated theories and methods. The articles below focus on these different levels and their special mediations, and propose new ways of viewing what takes place there.

Each of the following papers presents an individual case study. The point is not only (or even primarily) to argue that one should place students in similar circumstances to promote desired outcomes, but to present a persuasive example of how one might view collaborative learning taking place within such computer-supported contexts. In doing so, the authors propose intriguing extensions to theories that are important to CSCL, such as distributed cognition, discourse analysis, tacit knowledge, activity theory, and temporal analysis.

The study by Ruth Kershner, Neil Mercer, Paul Warwick, and Judith Kleine Staarman of elementary students' use of interactive whiteboards during small-group collaborative discussions builds on the theory of distributed cognition, in which people think collaboratively, mediated by physical and linguistic artifacts. It also applies Mercer's approach to discourse analysis - differentiating disputational, cumulative, and exploratory forms of children's group talk - for looking closely at sequential interaction. The paper uses the discourse analysis results to extend the theory of distributed cognition with the metaphor of a shared dynamic dialogic space as the focal point of the children's collective reasoning and co-construction of knowledge. The specific functions of the interactive whiteboard, combined with 'talk rules' instilled by the teacher, help to structure the dialogic space in which shared knowledge is co-constructed by the student groups.

The shared dynamic dialogic space — sometimes called the 'joint problem space' — could provide a new way of thinking about how the various critical dimensions of CSCL interactions come together. The interactive whiteboard, for instance, acting as a location for focusing shared attention on the group task, as a referential center for exploratory talk, as an external memory, common ground, or indexical source for group cognition, and as a visual foundation for group identity demonstrates useful functionality for computer support of collaborative knowledge building.

Folk theories and rationalist philosophies assume that knowers can unproblematically state knowledge explicitly. Knowledge is conceived of as a possession of individuals' minds, much like propositions stored in a computer database. But much of our knowledge as people, groups, and communities that carry out practical activities in the world is tacit, 
implicit, taken-for-granted, unstated. Meng Yew Tee and Dennis Karney investigate how tacit knowledge can be co-constructed, shared, and developed in a CSCL context. They look at how tacit knowledge of corporate culture surfaces in an online discussion of business management and how tacit hands-on know-how is built through role playing and the use of simulation games. Their analysis of student discourse suggested four key processes: socialization, externalization, combination, and internalization-consistent with Nonaka's model of knowledge creation. The authors stress the importance of viewing these processes in a situated way. The exploration of tacit knowledge as the oft over-looked foundation of collaborative learning provides an important corrective in post-cognitive theory to the concentration on explicit knowledge in rationalist, cognitivist, and folk theories.

The paper by Sinem Siyahhan, Sasha A. Barab, and Michael Downton focuses on another dimension that is generally overlooked by folk theories: that of social norms, particularly their influence on how people position each other's roles during interaction. This study looks at parent-student dyads playing an educational video game together after school. The analysis is framed in terms of Activity Theory, which includes among the mediations of goal-directed activity the dimensions of social norms, community, and the division of labor, in addition to mediation by artifacts (tools, symbols, technologies). The dialectic of roles was particularly interesting in this experimental context because the standard norms concerning parent-child relations interacted with the fact that the children were sometimes more expert at video-game operation and that the parents often positioned their children to take the lead during the 'educational' phases of the game. This opened up a space for productive exploration of the parent-child relationship by the participants.

The final paper of ijCSCL volume 5 addresses the temporal dimension of CSCL interaction. Folk theories of learning focus on the content and how it changes from some initial to some final state. However, to understand how collaborative learning takes place, we need to study how things gradually unfold during the period being studied. In particular, traditional theories conceive of time as an objective, smooth succession of moments. The Bakhtinian analysis by Maria Beatrice Ligorio and Giuseppe Ritella, however, treats the temporality experienced by the group in the classroom as a co-constructed encounter incorporating significant meaning and expression. Borrowing terminology from musicwhere the human experience of temporality is carefully orchestrated - the authors characterize phases of classroom interaction as proceeding with a tempo of adagio, andante, or allegretto. They see the dimension of temporality and the pace of sequential interaction as constructed by the responses of students to each other under the specific conditions of the classroom and its technology. The three chronotypes correspond to different modes of collaboration, in coordination with the sense of space that is simultaneously established.

The articles in this issue move far beyond folk theory and push the existing theories that have been popular in CSCL literature to better reflect the characteristics of interaction in CSCL settings. They suggest that computer-supported collaborative learning opens up a multi-dimensional shared world in which participants interact with each other, situated within an evolving context that they co-create. Knowledge, roles, space, and time are not simple givens whose characteristics can be assumed; they must be studied in each case through detailed analysis of the situated interaction. Of course, it is not necessary to address these theoretical frontiers of CSCL in every paper that claims to make significant and useful contributions to CSCL research; many research questions can be fully and rigorously pursued within the boundaries of established perspectives. IjCSCL welcomes both kinds of studies, those that make appropriate use of traditional frameworks and those that explore the boundaries of those frameworks. 
As we close the fifth year of production of ijCSCL, we would like to extend our gratitude to the many people who have supported the journal as Editorial Board members, authors, subscribers, and readers. In particular, we thank the following CSCL researchers who reviewed submissions to the journal; their committed and expert volunteer work is the single most important factor in maintaining the high quality of ijCSCL as a leading international journal and as the venue of choice for CSCL research:

Shaaron Ainsworth, Rick Alterman, Jerry Andriessen, Hans Christian Arnseth, Gerardo Ayala, Michael Baker, Maria Bannert, Liam Bannon, Daniel Bodemer, Jacqueline Bourdeau, Paul Brna, Bertram Bruce, Amy Bruckman, Juergen Buder, Murat Cakir, John Carroll, Carol Chan, Tak-Wai Chan, Rosanna Chan, Elizabeth Charles, Cesar Collazos, Ulrike Cress, Charles Crook, Lucilla Crosta, Ton de Jong, Anne Deiglmayr, Sharon Derry, Pierre Dillenbourg, Angelique Dimitrakopoulou, Lone Dirckinck-Holmfeld, Paul Dourish, Nathan Dwyer, Noel Enyedy, Frank Fischer, Brian Foley, Andrea Forte, Hugo Fuks, Sean Goggins, Ricki Goldman, Jonathan Grudin, Frode Guribye, Joerg Haake, Kai Hakkarainen, Paivi Hakkinen, Andreas Harrer, Wu He, Thomas Herrmann, Friedrich Hesse, Cindy Hmelo-Silver, Christopher Hoadley, Ulrich Hoppe, Christine Howe, James Hudson, Diane Hui, Sanna Jarvela, Patrick Jermann, Richard Joiner, Christopher Jones, Regina Jucks, Yael Kali, Victor Kaptelinin, Manu Kapur, Fengfeng Ke, Diane Ketelhut, Andrea Kienle, Joachim Kimmerle, Paul Kirschner, Lars Kobbe, Matthew J. Koehler, Timothy Koschmann, Ingeborg Krange, Therese Laferriere, Minna Lakkala, Victor Lally, Mary Lamon, Johann Larusson, Nancy Law, Oskar Lindwall, Lasse Lipponen, Jacques Lonchamp, Chee-Kit Looi, Jingyan Lu, Rose Luckin, Sten R. Ludvigsen, Andreas Lund, Kristine Lund, Johan Lundin, Alejandra Martinez, Richard Medina, Naomi Miyake, Anders Morch, Johannes Moskaliuk, Daisy Mwanza-Simwami, Bonnie Nardi, Brian Nelson, Bernhard Nett, Matthias Nuckles, Angela O'Donnell, Hiroaki Ogata, Claire O’Malley, Jun Oshima, Roy Pea, Ruediger Pfister, Ingvill Rasmussen, Janet Read, Peter Reimann, Ann Renninger, Jochen Rick, Tim Roberts, Jennifer Rode, Markus Rohde, Jeremy Roschelle, Carolyn Rose, Liam Rourke, Nikol Rummel, Nadira Saab, Roger Saljo, Johann Sarmiento-Klapper, Tammy Schellens, Gregg Schraw, Baruch Schwarz, Anna Sfard, David Shaffer, Wesley Shumar, Amy Soller, Nancy Songer, Hans Spada, Marc Stadtler, Constance Steinkuehler, Jan-Willem Strijbos, Masanori Sugimoto, Daniel Suthers, Berthel Sutter, Gustav Taxen, Pierre Tchounikine, Chris Teplovs, Ramon Prudencio Toledo, Stefan Trausan-Matu, Jan van Aalst, Ravi Vatrapu, Marjaana Veermans, Barbara Wasson, Jim Waters, Rupert Wegerif, Armin Weinberger, Gordon Wells, Martin Wessner, Tobin White, Volker Wulf, Fatos Xhafa, Ling Ling Yen, Jennifer Yeo, Joyce Yukawa, Coco Zhao, Nan Zhou.

\section{$* * *$}

Start planning now for the up-coming CSCL 2011 conference in Hong Kong, July 48 (see: http://www.isls.org/cscl2011). Hong Kong is an exciting crossroads of the world, a bridge between East and West. It is easily accessible from Europe and the Americas by direct flights. The culinary capital of Canton, it offers hotels for every budget. The conference venue at the University of Hong Kong - one of Asia's premier universitiesis located within walking distance of the heart of Hong Kong. A former British colony, Hong Kong uses English widely. CSCL 2011 is designed to offer an affordable global experience, including a post-conference group tour of educational and tourist sites in mainland China July 10-15-bring your family and students. We look forward to seeing you there. 